Morales Bueno, P. (2018). Aprendizaje basado en problemas (ABP) y habilidades de pensamiento crítico ¿una relación vinculante? Revista Electrónica Interuniversitaria de Formación del Profesorado, 21(2), 91-108.

\title{
Aprendizaje basado en problemas (ABP) y habilidades de pensamiento crítico, ¿una relación vinculante?
}

\author{
Patricia Morales Bueno \\ Pontificia Universidad Católica del Perú PUCP
}

\section{Resumen}

La propuesta metodológica Aprendizaje Basado en Problemas (ABP) se ha convertido en una alternativa atractiva para ejecutar los cambios en los modelos educativos de la educación superior, en particular porque su entorno de aprendizaje es propicio para el desarrollo de habilidades de pensamiento de orden superior, como el pensamiento crítico. Sin embargo, este proceso no ocurre de manera espontánea, es necesario tomar en consideración los aspectos del modelo que pueden influir en el nivel de logro cuando se tiene el objetivo de mejorar estas habilidades en la formación universitaria. En este trabajo se analiza los aspectos más importantes de la metodología ABP y de la enseñanza de habilidades de pensamiento crítico, buscando poner en evidencia los aspectos más sensibles que afectan la relación vinculante entre ellos. Se discute el caso de las modalidades híbridas ABP y se muestran algunos resultados de investigación que sustentan la discusión.

\section{Palabras clave}

ABP; pensamiento crítico; habilidades de pensamiento de orden superior; enseñanza universitaria.

\section{Problem-based learning (PBL) and critical thinking skills - a binding relationship?}

\section{Abstract}

Problem Based Learning ( $\mathrm{PBL}$ ) has become an attractive alternative to implement changes in the educational models of higher education, particularly because its learning environment is auspicious for the development of higher order thinking skills, as critical thinking. However, this process does not occur spontaneously, it is necessary to take into 
account the aspects of the model that can influence the level of achievement when the goal is to improve these skills in university education. This paper analyzes the most important aspects of the PBL methodology and the teaching of critical thinking skills, seeking to highlight the most sensitive aspects that affect the binding relationship between them. The case of hybrid PBL models is discussed and some research results that support the discussion are shown.

\section{Key words}

PBL; critical thinking; higher order thinking skills; higher education.

\section{Introducción}

Sin duda, el interés por la innovación educativa ha crecido considerablemente en las últimas décadas y, en particular, en la educación superior. Muchas instituciones universitarias han replanteado sus modelos educativos con el propósito de garantizar que sus egresados cuenten con las competencias que les permitirán incorporarse a su entorno laboral y aportar a la solución de los diferentes problemas de la sociedad. Un aspecto destacable en este proceso de transformación es la especial atención que ha tomado el desarrollo y fortalecimiento de las llamadas competencias genéricas, a la par de aquellas vinculadas específicamente a la disciplina o profesión. Es evidente la importancia de incorporar al modelo educativo el desarrollo de habilidades que permitan al nuevo profesional enfrentar con éxito diversas situaciones, en las que deberá por ejemplo, demostrar capacidad para elaborar juicios a partir del análisis de un conjunto de hechos o evidencias o, tomar decisiones sobre la base del análisis e integración de los diferentes aspectos que pueden estar implicados en una problemática particular. Esta capacidad está asociada directamente al desarrollo y uso de habilidades de pensamiento de orden superior, cabe entonces plantear la interrogante ¿de qué manera se puede propiciar el desarrollo de estas habilidades en el modelo educativo?

Existe un importante consenso de opiniones que sugiere que para promover la mejora de las habilidades de pensamiento de orden superior es necesario crear un ambiente de aprendizaje propicio para que los estudiantes asuman un rol activo en el proceso. Esto supone que ellos puedan explorar directamente situaciones problemáticas complejas por medio del planteamiento de preguntas abiertas que guíen la discusión, el intercambio de ideas y la construcción de significados. Es importante además el aspecto social del proceso de aprendizaje y, por ello, se promueve el desarrollo de actividades grupales que brinden oportunidades para que los estudiantes puedan reflexionar acerca de sus propios procesos de razonamiento, además de establecer las conexiones y la aplicabilidad entre el contenido del aprendizaje logrado y la vida real. De esta manera se establece la necesidad de un cambio desde un modelo educativo tradicionalmente centrado en el contenido, a otro centrado en el aprendizaje, en donde el estudiante es el actor principal (Albaaly, 2012; Marshall \& Horton, 2011; Miri, David \& Uri, 2007; Snyder \& Snyder, 2008).

La propuesta metodológica Aprendizaje Basado en Problemas (ABP) (Barrows, 1996) se ha convertido en una alternativa atractiva para ejecutar los cambios en los modelos educativos de la educación superior. Su efectividad está condicionada a que sea asumida con las implicancias conceptuales referentes al cambio de roles del docente y del estudiante, así como con la inserción del proceso de enseñanza aprendizaje en el contexto complejo de la realidad en la cual se desarrolla y el reconocimiento de la naturaleza cambiante del conocimiento. El contexto de aprendizaje propiciado por el ABP permite el desarrollo de 
habilidades no consideradas en los métodos tradicionales, como son, el trabajo colaborativo en equipos, la capacidad investigativa, el aprendizaje autónomo y las habilidades de pensamiento crítico (Biggs, 2005; Boud \& Feletti, 1997; Duch, Groh \& Allen, 2001; Hmelo-Silver \& Ferrari, 1997; Wilkerson \& Gijselaers, 1996).

La transición desde un currículum tradicional hacia un currículum ABP no es un proceso sencillo. Es necesario que todos los componentes involucrados: políticas educativas de la institución, currículum, aspectos administrativos, concepciones sobre la enseñanza y el aprendizaje de profesores y estudiantes, entre otros, estén involucrados en esta transformación. El nivel de aproximación hacia un modelo ABP puro, depende principalmente del grado de autonomía que se le permita tener al estudiante en cuanto a su propio proceso de aprendizaje (Guerra y Kolmos, 2011). En función de esta situación surgen las llamadas modalidades híbridas, en las cuales se combinan algunos aspectos de la metodología original con elementos que aún conservan características más convencionales.

Cuando el modelo se adopta con limitaciones, ya sea pedagógicas, administrativas o institucionales, la consecuencia inmediata es incrementar el nivel de control y andamiaje de los diseños de escenarios y procesos. Esto significa que el nivel de autonomía del estudiante disminuye y, fácilmente retornará a un rol más pasivo en el que difícilmente tendrá la disposición suficiente para desplegar sus habilidades de razonamiento de orden superior. Por ello, se hace necesario cuestionar ¿cuáles son las dimensiones de la enseñanza de habilidades del pensamiento que se ven favorecidas en un contexto ABP? ¿De qué manera la modalidad de implementación del ABP influye en el desarrollo de las habilidades de pensamiento crítico?

En las siguientes secciones se hará una revisión de estos aspectos, mostrando las características de la metodología que podrían considerarse sensibles para lograr de manera efectiva el desarrollo del pensamiento crítico, complementando la discusión con algunos resultados de investigación.

\section{Características del ABP}

El Aprendizaje Basado en Problemas es una visión educativa que promueve el aprendizaje abierto, reflexivo y crítico, con un enfoque holístico del conocimiento que reconoce su naturaleza compleja y cambiante, e involucra a una comunidad de personas que interactúan en colaboración para tomar decisiones en relación a diferentes situaciones problemáticas que deben enfrentar. En este sentido, el $A B P$ es el medio por el cual se hace posible establecer las condiciones que conducen al aprendizaje activo, contextualizado, integrado y orientado a la comprensión, brindando oportunidades para reflexionar sobre la experiencia educativa y aplicar lo aprendido. Por lo tanto, está claro que los objetivos de aprendizaje van más allá de la mera adquisición de contenido disciplinario (Engel, 1997; Hmelo-Silver, 2004; Margetson, 1997).

El contexto de aprendizaje en la metodología ABP promueve la interacción de diferentes factores que involucran a estudiantes y profesores, pero manteniendo el rol central en los primeros. El docente no es responsable de entregar información, sino que dirige y guía al alumno en su búsqueda, brindando oportunidades para desarrollar habilidades de aprendizaje y pensamiento de orden superior, contribuyendo así a la formación de estudiantes autónomos, que es uno de los principales objetivos de la educación universitaria.

Con frecuencia se ubica el origen del ABP, en la década de los 1970's, en la Escuela de Medicina de la Universidad McMaster (Canadá) y se reconoce a Howard Barrows como uno 
de sus representantes más emblemáticos. El modelo fue rápidamente adoptado por otras escuelas de medicina y posteriormente por muchas disciplinas, programas e instituciones. Las bases teóricas del ABP han sido analizadas y discutidas con mayor profundidad a partir de los 1990's, sin embargo no hay una posición unánime respecto a ellas, lo que ha ocasionado que en la literatura el modelo haya sido definido y descrito en una diversidad de formas (Gijbels, Dochy, Van den Bossche y Segers, 2005; Graaff \& Kolmos, 2003; Savery, 2006). La variedad de modalidades de adopción de la metodología está condicionada por la naturaleza del dominio y de los objetivos específicos de los programas en los que se adscribe. Sin embargo, se hace necesario formular una definición básica o un modelo central que sirva de base de comparación con otros métodos educativos y, para ello, la referencia más frecuente es Howard Barrows, quien describe las características centrales del modelo de la siguiente manera (Barrows, 1996; Hmelo-Silver \& Barrows, 2006):

- El ABP es un enfoque centrado en el estudiante, quien es el que determina lo que necesita aprender. Corresponde a los alumnos identificar los aspectos clave de los problemas que enfrentan, definir sus necesidades de conocimiento y emprender la búsqueda del conocimiento faltante.

- El aprendizaje se produce en grupos pequeños de estudiantes, que llegan a conformar una pequeña comunidad de investigación, en donde los participantes se escuchan entre sí, están abiertos a diferentes puntos de vista y pueden trabajar en colaboración para llegar a conclusiones razonables.

- Los problemas que inician el proceso se presentan desestructurados y con un cierto nivel de incertidumbre para promover que los estudiantes pongan en práctica no solo múltiples razonamientos vinculados a las posibles causas del problema, sino también múltiples razonamientos relacionados a la forma de resolverlo.

- La selección de problemas debe realizarse en función de su autenticidad, lo que implica que estén alineados a la práctica profesional o al mundo real.

- Los profesores actúan como facilitadores y tutores planteando a los estudiantes los tipos de preguntas metacognitivas que esperan que los estudiantes aprendan a formularse a sí mismos, de tal manera que la intervención del tutor sea cada vez menor.

La adopción del ABP en las diferentes instituciones, facultades y disciplinas, se ha realizado mediante un proceso de adaptación a las características propias, contextos y necesidades de cada una de ellas, por lo que podría decirse que hay tantas modalidades de ABP como implementaciones reportadas. Savin-Baden (2000) describe cinco modelos ABP en función de los objetivos de aprendizaje que guían el diseño del escenario problemático correspondiente. Estos van desde objetivos específicos acotados al contenido de una asignatura, hasta objetivos abiertos al conocimiento interdisciplinario, necesario para el análisis y la solución de problemas. El diseño de los problemas varía desde escenarios restringidos a respuestas correctas, hasta escenarios desestructurados y de respuesta abierta. Es así que el modelo puede ser adoptado en la institución como un enfoque holístico o parcial del currículum, aproximándose más cercanamente al modelo original de McMaster, denominado también la forma pura del ABP. La otra opción, que es bastante frecuente, es la adopción como estrategia o técnica didáctica en uno o varios módulos de disciplinas o asignaturas aisladas pertenecientes a un currículum convencional.

La literatura señala como principales metas de la adopción del ABP en la educación superior, las siguientes (Duch et al., 2001; Hmelo-Silver, 2004): 
- Desarrollo de habilidades para la resolución de problemas, tanto en la adquisición como en la aplicación del conocimiento en diferentes situaciones.

- Desarrollo del pensamiento crítico y de la capacidad de analizar y resolver problemas complejos del mundo real.

- Desarrollo de habilidades para la búsqueda, evaluación y uso de recursos de aprendizaje apropiados.

- Desarrollo de habilidades, valores y actitudes para el trabajo colaborativo en equipos y grupos pequeños

- Desarrollo de habilidades para el aprendizaje autónomo, autodirigido y continuo.

El ABP no es un programa específico para la instrucción del pensamiento, sin embargo, sus objetivos y metas guardan una alta coincidencia con los de este tipo de programas. El proceso seguido en el modelo ABP promueve que el estudiante desarrolle habilidades relacionadas con identificar situaciones problemáticas, plantearse preguntas, investigar, sustentar razonadamente sus ideas, contrastarlas con las de otros, reformular el problema y sus estrategias para abordarlo, formular conclusiones y juicios de manera razonada y reflexiva, entre otras (Hmelo-Silver \& Ferrari, 1997). Todas estas habilidades, están incluidas en la mayoría de modelos para la enseñanza del pensamiento crítico (Halpern, 1989; Facione, 2000; Ennis, 1996; Facione, 2011).

\section{Pensamiento crítico}

Cuando se habla de las habilidades de pensamiento de orden superior, comúnmente se alude a un tipo de pensamiento no-algorítmico, esforzado, complejo, que permite elaborar juicios y múltiples soluciones a problemas, normalmente comprendidos en un contexto con un cierto nivel de incertidumbre (Hmelo-Silver \& Ferrari, 1997). Newman (1990) propone una distinción entre las habilidades de pensamiento, denominando de orden inferior a aquellas que demandan únicamente aplicación mecánica o rutinaria de información adquirida previamente. En el otro extremo, las de orden superior son las habilidades que desafían al estudiante a interpretar, analizar o manipular información. Su uso se ubica en una tercera dimensión de reflexión sobre el conocimiento, en la que el estudiante analiza y elabora juicios acerca de la situación problemática que motivó el aprendizaje. Esta dimensión se apoya en otras dos: adquisición y aplicación de conocimiento, en las que se despliegan principalmente habilidades básicas o de orden inferior (Phan, 2009). Normalmente se incluye en la categoría de habilidades de pensamiento de orden superior al pensamiento crítico, lógico, reflexivo, metacognitivo y creativo.

\section{Concepto de pensamiento crítico}

Desde el enfoque de la psicología cognitiva y evolutiva, así como de las teorías de la inteligencia, el interés hacia el proceso de pensamiento se sustenta en la investigación empírica, en las distinciones entre expertos y novatos en el aprendizaje de ideas complejas y, en la relación del pensamiento crítico con la resolución de problemas. D. Halpern (1989), una de las principales representantes de esta perspectiva, se refiere al pensamiento crítico como aquel que tiene un propósito, es razonado y dirigido a metas. Es la clase de pensamiento involucrado en la resolución de problemas, la formulación de inferencias, el cálculo de probabilidades y la toma de decisiones e, involucra además un componente evaluativo en el que se evalúan los logros de nuestros procesos de pensamiento y el proceso mismo. El pensamiento es entonces dirigido, pues se focaliza en obtener un logro 
deseado. Un pensamiento que no tiene un propósito y no incorpora una evaluación consciente no puede considerarse crítico.

Un aspecto importante a considerar se relaciona con la actitud o disposición del pensamiento. Los buenos pensadores están motivados y dispuestos a realizar el esfuerzo consciente necesario para trabajar de una manera planificada, buscar la exactitud, recopilar información y tener persistencia cuando la solución no es muy evidente o requiere varias etapas (Halpern, 1989; Facione, 2000). Para Halpern, si las habilidades inherentes al pensamiento crítico pueden ser mejoradas con la enseñanza, el objetivo de ésta debería ser que los estudiantes puedan transferir estas habilidades al mundo real, fuera de las situaciones de la sala de clases. La meta es promover el aprendizaje de habilidades de pensamiento transcontextuales y conscientes, que permitan dirigir el propio pensamiento y aprendizaje. En este sentido, propone un modelo para la enseñanza de las habilidades del pensamiento que permite su transferencia a través de los dominios del conocimiento y que consiste de cuatro partes: el componente actitudinal o de la disposición; la instrucción y la práctica de las habilidades del pensamiento crítico; actividades de capacitación en el uso de estructuras para facilitar la transferencia a través de los contextos y, el componente metacognitivo usado para dirigir y evaluar el pensamiento (Halpern, 1989, 1998).

En la línea de Halpern y otros como Ennis (1996), Facione (2011) y Walton (2006), C. Saiz y S.F. Rivas, investigadores de la Universidad de Salamanca (España), desarrollaron un programa denominado ARDESOS (Argumentación/Decisión/Solución/Salamanca) (Saiz y Rivas, 2011, 2012). El concepto teórico que subyace a este programa asume que pensar críticamente implica reflexión y acción para alcanzar nuestras metas. Pensar críticamente es razonar y tomar decisiones para resolver problemas lo más efectivamente posible. ARDESOS es un programa independiente de un dominio, de enseñanza directa de habilidades consideradas esenciales para el desarrollo del pensamiento crítico. La estrategia de enseñanza-aprendizaje empleada es el ABP. Los estudiantes, organizados en grupos, participan en actividades que involucran la reflexión y discusión acerca de situaciones problemáticas previamente diseñadas para promover habilidades de pensamiento crítico. El método estimula los procesos metacognitivos y permite que los estudiantes pongan en práctica sus habilidades, desafiándolos con situaciones reales, en donde deben buscar e investigar sus propias respuestas y soluciones.

Las características comunes de estos modelos de enseñanza de pensamiento crítico incluyen el uso de estrategias en las que el estudiante se involucre activamente, siendo el aprendizaje el centro del proceso y no solamente el contenido. Además, las modalidades de evaluación son coherentes con la estrategia seguida, es decir se emplean recursos que desafíen intelectualmente al estudiante en vez de reducirse a un ejercicio de memoria o repetición (Snyder \& Snyder, 2008). El ABP tiene como característica principal el iniciar el proceso de aprendizaje de los estudiantes con la presentación de un problema desafiante correspondiente a una situación real o realista. Se espera que al abordarlo, el estudiante tenga la oportunidad de desarrollar un conjunto de habilidades que incluyen a las del pensamiento crítico ¿cuáles son las condiciones que propician que se establezca esta relación vinculante?

\section{ABP y pensamiento crítico}

Dos componentes cruciales para promover el desarrollo de habilidades de pensamiento crítico en la metodología ABP son el diseño del problema y el desarrollo del proceso. 


\section{El problema}

El escenario o problema ABP constituye el reto inicial y motivador del proceso de aprendizaje y, como tal, debe cumplir con un conjunto de características que aseguren el involucramiento del estudiante en la construcción de su aprendizaje y el despliegue de las habilidades necesarias para proponer y sustentar propuestas de solución. El modelo 3 C3R, propuesto por Hung (2006), grafica muy claramente los aspectos a considerar en el diseño de un problema ABP (ver Figura 1).

\section{Investigación}

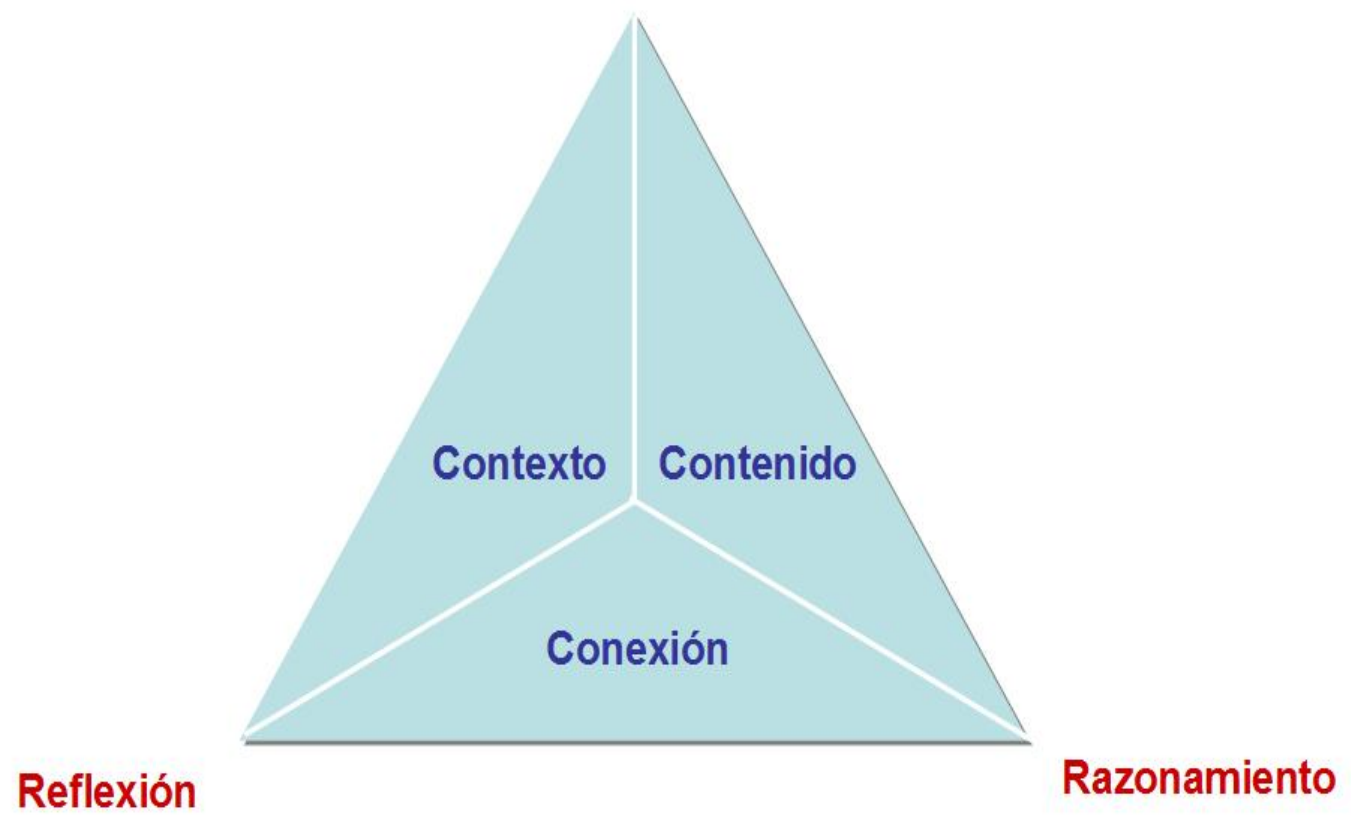

Figura 1. Modelo 3C3R para el diseño de problemas ABP (Hung, 2006)

Los componentes centrales del modelo: contenido, contexto y conexión, tienen como funciones principales establecer las bases del problema, de tal manera que guarden relación directa con las metas y objetivos de aprendizaje previstos; contextualizar el dominio de conocimiento $\mathrm{y}$, además, guiar a los estudiantes a construir marcos conceptuales integrados.

El componente de contenido debe asegurar la alineación apropiada entre el alcance del problema y los contenidos curriculares en amplitud y profundidad. El alcance debe ser ajustado adecuadamente para que el aprendizaje del estudiante se produzca de acuerdo al objetivo trazado, en cuanto al área de conocimiento y a las habilidades intelectuales necesarias para alcanzarlo. Debe haber un balance entre el nivel de profundidad, complejidad y desestructuración del problema. Si para resolver un escenario propuesto se requiere únicamente de información básica, la necesidad y motivación del estudiante para profundizar en el tema desaparecen. Cuanto mayor desestructuración tenga el problema se multiplicarán los mecanismos de razonamiento y las alternativas de solución y, con ello, se brindará al estudiante la oportunidad de explorar diferentes alternativas de análisis. En consecuencia, la comprensión del dominio será más profunda. 
El contexto asegura que la situación planteada sea auténtica (real o realista) y además relevante para el área profesional en la que se forma el estudiante, sus intereses personales o su vida como miembro de la sociedad. La singularidad de un escenario real o realista desencadenará diferentes formas de razonamiento que el estudiante deberá tomar en cuenta. Para ello, es necesario que se preste cuidado en la validez del contexto en el que se sitúa el problema. Un escenario médico, por ejemplo, no sería válido para estudiantes de ingeniería civil.

El componente de conexión facilita que los estudiantes logren integrar el conocimiento e interconectar los conceptos de tal forma de establecer una red conceptual del tema. A menudo, el problema ABP está diseñado en etapas, que se irán planteando gradual y secuencialmente. Cada nueva etapa proporciona al estudiante información adicional que se deberá relacionar con las etapas anteriores y que le permitirá replantear su propuesta de solución. Así mismo, se promueve que el estudiante comprenda cómo los conceptos o variables pueden manifestarse en diferentes situaciones y evidenciar su interrelación.

Los componentes de procesamiento: investigación, razonamiento y reflexión, facilitan la implicación consciente y significativa del estudiante en su proceso de aprendizaje. Son los elementos dinámicos del modelo, ya que actúan como activadores para que los estudiantes obtengan provecho de los componentes centrales, además de regular el nivel de los procesos cognitivos de acuerdo a sus capacidades. La investigación activa los componentes centrales, promueve el desarrollo de habilidades para la búsqueda y procesamiento de información, facilita las conexiones conceptuales durante el aprendizaje del estudiante y soporta los procesos de razonamiento y reflexión. El componente de razonamiento promueve la aplicación del conocimiento adquirido a partir de la investigación y el desarrollo de habilidades para la solución de problemas. El estudiante profundiza y expande su comprensión conceptual. Los procesos de investigación y razonamiento ocurren de manera simultánea y reiterativa y se complementan mutuamente. Las actividades cognitivas involucradas en estos dos procesos son habilidades de pensamiento de orden superior.

En la Figura 2 se representan diferentes ajustes de nivel de las habilidades de investigación y de razonamiento que pueden darse en el diseño de un escenario ABP. Los casos que se muestran corresponden a situaciones extremas de bajo o alto nivel de habilidad y sus posibles combinaciones. Los problemas altamente desestructurados y con poca información acerca del conocimiento necesario para abordarlos se ubican en el caso en el que los estudiantes deben desplegar un alto nivel de habilidades para la investigación y para el razonamiento. Por el contrario, los escenarios que presentan casos específicos para situar el problema, en donde es evidente el contenido conceptual que debe aplicarse para proponer soluciones que normalmente tienen un rango acotado de alternativas, corresponden a situaciones en donde tanto el nivel de habilidades de investigación como de razonamiento requeridos son bajos.

El componente de reflexión actúa como un elemento guía para la metacognición, optimiza el proceso ABP asegurando el máximo efecto de los otros componentes. La reflexión ayuda a los estudiantes no solamente a integrar lo aprendido, sino que va más allá del alcance del problema y cultiva en los estudiantes el hábito de desarrollar y utilizar sus habilidades para el aprendizaje auto-dirigido y para toda la vida. 


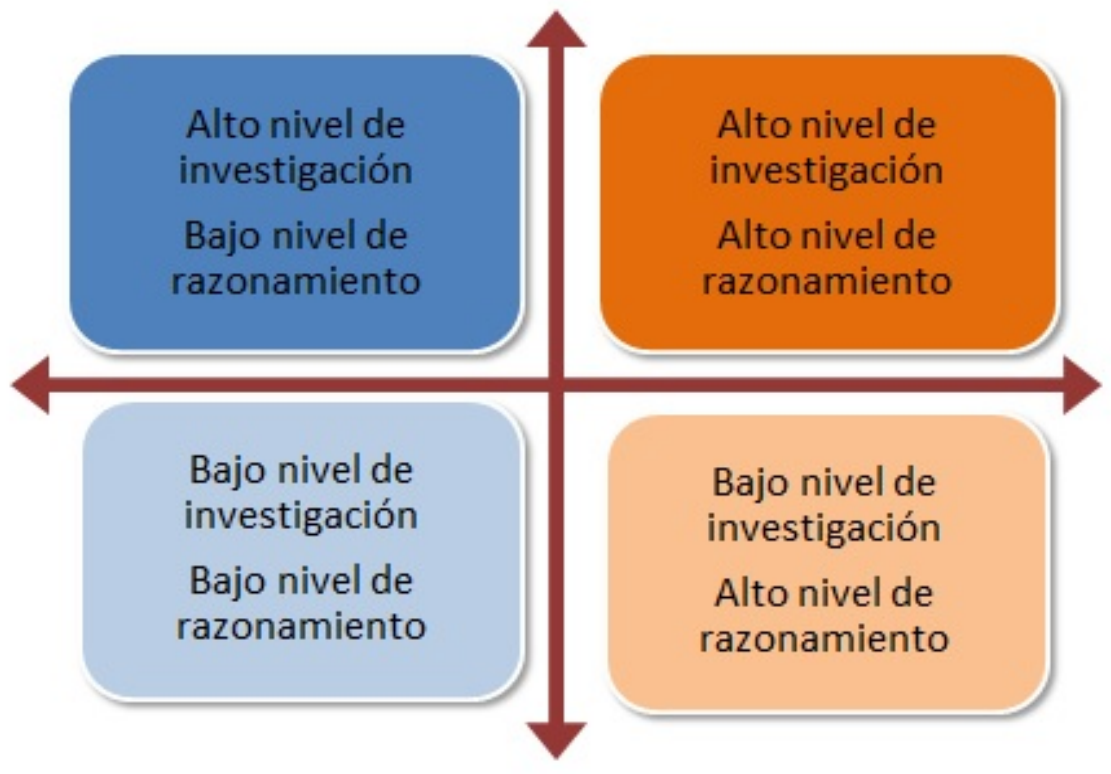

Figura 2. Interacción de los componentes de investigación y razonamiento en el modelo 3C3R (adaptado de Hung (2006))

\section{El proceso}

El proceso se inicia con la presentación del problema a los estudiantes. Se espera que ellos organicen sus ideas con respecto a la situación planteada, que identifiquen su naturaleza y los factores o aspectos involucrados en ella. Luego de una primera lluvia de ideas, los grupos de estudiantes las analizan de forma crítica, para identificar los aspectos que se relacionan con sus conocimientos previos sobre el tema, así como los aspectos que no entienden y para los cuales necesitan adquirir nuevos conocimientos.

En la siguiente etapa, los estudiantes se organizan para buscar, revisar y sistematizar la nueva información. Es aquí donde se presentan algunas diferencias en la forma de implementación del ABP. Según la naturaleza y objetivos del curso, el número y nivel académico de los estudiantes, los recursos disponibles, entre otros aspectos; el diseño del proceso podrá considerar un camino más cercano al modelo original de McMaster, en donde el trabajo de los grupos de estudiantes se realiza de una forma más autónoma, siendo el rol del profesor el de un tutor o consultor. Otras alternativas implican una mayor intervención del profesor, en las denominadas modalidades híbridas. Estas aproximaciones son más sensibles a los factores externos (institucionales, administrativos, curriculares) y ello puede afectar al logro de los objetivos esperados, especialmente en el desarrollo de habilidades de pensamiento de orden superior. Por lo general, las modalidades híbridas conservan el componente de trabajo en grupos colaborativos, el uso de escenarios (con mayor o menor grado de complejidad y estructuración) y el rol de facilitador del profesor de manera parcial, limitándose a algunos momentos del proceso ya que mantiene el control en cierto grado. Comúnmente, el profesor estructura el proceso de aprendizaje a través de actividades individuales o grupales pre-diseñadas, demostraciones en aula, clases expositivas, etc. 
En la última etapa, el grupo aplica el nuevo conocimiento al problema inicial, verificando si sus primeros planteamientos pueden ser reordenados o redefinidos en función de lo aprendido. Luego de ello estarán en capacidad de formular y argumentar sus propias propuestas de solución.

El pensamiento crítico no es una habilidad innata, requiere de aprendizaje, entrenamiento y práctica. El entorno de aprendizaje en el ABP es propicio para cultivar y fortalecer estas habilidades, por lo que estos objetivos deben formar parte intencionada en el proceso. En ese sentido, el docente cumple el rol de un aprendiz experto con la capacidad de modelar buenas estrategias para el aprendizaje y el razonamiento (Hmelo-Silver \& Ferrari, 1997). Las estrategias propuestas para la enseñanza de habilidades de pensamiento crítico sugieren comúnmente crear el hábito en el aprendiz de plantearse interrogantes de tipo metacognitivo. Al inicio del proceso, el docente modela esta práctica proponiendo el tipo de preguntas que se espera el estudiante pueda plantear por sí mismo en algún momento. Por ejemplo: ¿qué preguntas podrías plantear frente a esta situación? ¿Cuál piensas que es el problema en este caso? ¿Por qué razón optas por esa alternativa? ¿Cuáles son las implicancias de la situación? ¿Cómo estás enfocando el tema? ¿Podría enfocarse de una manera distinta? ¿Estás de acuerdo con lo que ha explicado tu compañero? Conforme el estudiante vaya ganado experiencia en esta práctica, la intervención del docente debe disminuir. Es en este momento en que el trabajo colaborativo en grupos cobra especial relevancia, pues permite que los estudiantes puedan comparar y compartir sus estrategias de razonamiento con sus pares, mejorando así conjuntamente sus habilidades (Hmelo-Silver \& Ferrari, 1997; Snyder \& Snyder, 2008).

Facione (2007) analiza de manera vinculante las habilidades para la solución de problemas y las de pensamiento crítico y propone una estrategia de 5 etapas, que las resume con el nombre de IDEAS (Identify, Determine, Enumerate, Assess, Scrutinize). En la primera etapa se identifica el problema y se establecen prioridades para abordarlo. El estudiante tiene ocasión de utilizar sus habilidades para interpretar situaciones, datos, procedimientos, etc. Luego determina la información relevante, define el contexto que enmarca el problema y profundiza la comprensión del mismo. Para ello, el estudiante debe esbozar sus primeras hipótesis, asegurando los elementos necesarios para llegar a conclusiones razonables en relación al problema. Con mayor claridad, en la siguiente etapa se enumera las rutas alternativas para buscar una solución, haciendo uso de las habilidades para identificar relaciones entre las diferentes piezas de información disponibles. A continuación se recurre a las habilidades de análisis y evaluación para poder examinar las alternativas propuestas y tomar una decisión preliminar, la cual debe fundamentarse con argumentos construidos en base a las evidencias, al conocimiento involucrado, así como a los aspectos metodológicos y contextuales. Finalmente, se examina cuidadosamente todo el proceso y se auto-corrige lo que sea necesario, aplicando habilidades para monitorear conscientemente las propias actividades cognitivas. En la Figura 3 se representa el proceso ABP y las preguntas inherentes al proceso que pueden propiciar la puesta en acción de habilidades de pensamiento crítico. 


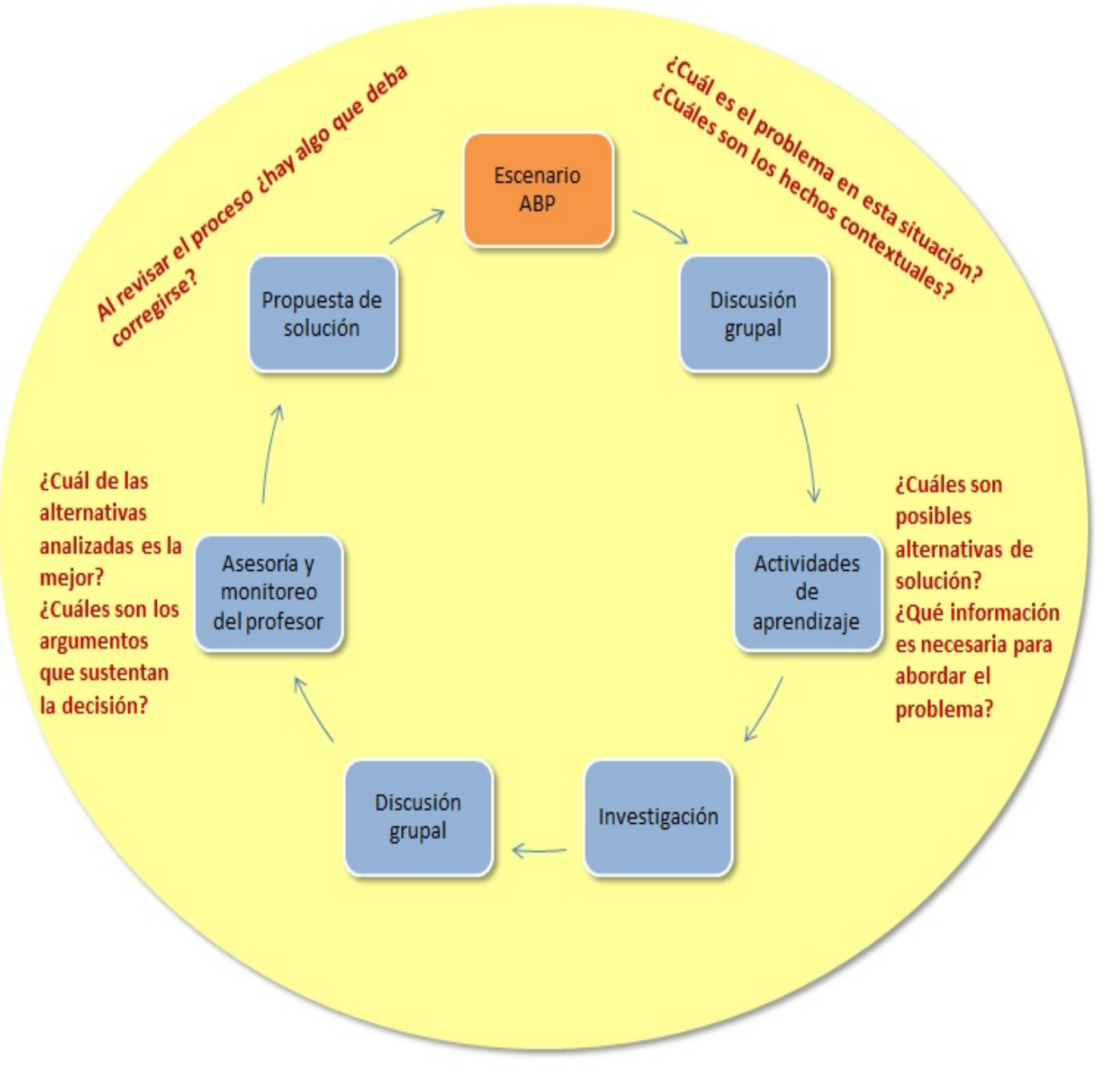

Figura 3. El proceso ABP y su relación vinculante con las habilidades de pensamiento crítico (elaboración propia)

\section{Algunos resultados de investigación}

La adopción de modalidades híbridas ABP es bastante frecuente, en ellas el grado de autonomía del estudiante depende del nivel de aproximación al modelo original. Las limitaciones de los modelos híbridos son mayores cuando se aplican en una asignatura específica en un currículum convencional. Nuestro interés se centró en determinar de qué manera influía el modelo de adopción de $A B P$ en el desarrollo de habilidades de pensamiento crítico. Para ello se tomó de referencia un modelo híbrido aplicado por varios años (2008 - 2016) en un curso de Química General de primer año de Ciencias e Ingeniería de una universidad peruana. Este modelo fue diseñado tomando en consideración que los estudiantes al inicio de su formación universitaria no mostraban mayor experiencia en el uso de habilidades para el aprendizaje autónomo, por tanto se mantuvo un nivel moderado de control del docente y un grado de moderado a alto en la estructuración del escenario ABP. Para cada unidad temática del curso se usaba un escenario relacionado estrechamente con el contenido, pero que involucraba que el estudiante realizara algunas actividades de investigación. El problema implicaba la posibilidad de optar por diferentes alternativas de solución y, por lo general, el estudiante debía tomar decisiones fundamentadas con argumentos al finalizar el proceso. Un esquema del modelo se muestra en la Figura 4. 


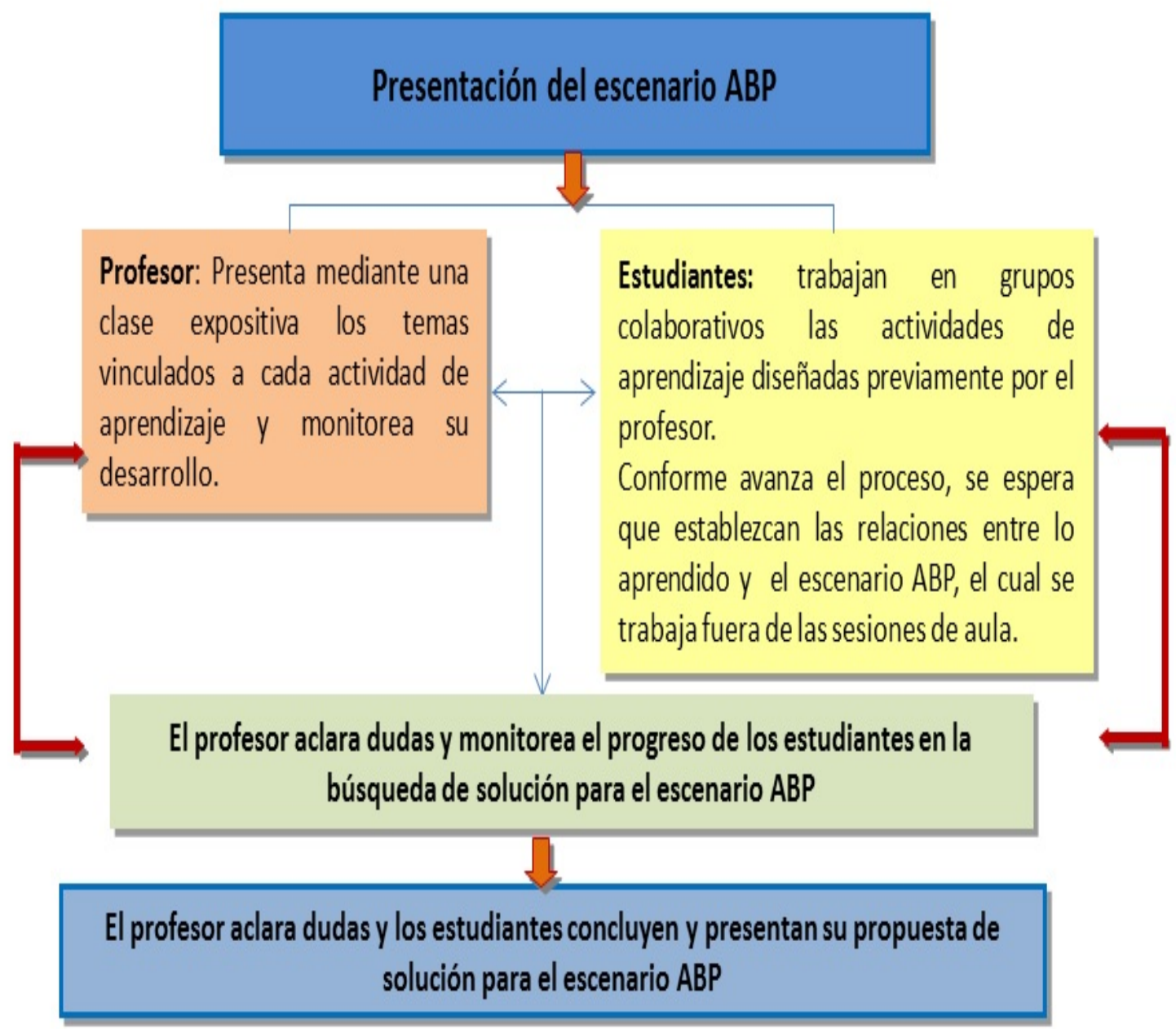

Figura 4. Modelo híbrido ABP aplicado en un curso de Química General de primer año de Ciencias e Ingeniería de una universidad peruana (elaboración propia)

En un primer estudio (Morales Bueno, 2016) se trabajó con tres grupos de estudiantes que llevaron el curso en los años 2010, 2011 y 2012 respectivamente. La edad promedio de los tres grupos era 18 años y su composición en función del sexo era $67.6 \%$ varones y $32.4 \%$ mujeres. La evaluación de las habilidades de pensamiento crítico se realizó mediante el test PENCRISAL (Rivas, Morales Bueno \& Saiz, 2014) que considera cinco dimensiones: razonamiento deductivo, razonamiento inductivo, razonamiento práctico, toma de decisiones y solución de problemas. El test fue aplicado al finalizar el semestre en cada caso. En la Figura 5 se muestra los resultados obtenidos, expresados como porcentaje del puntaje máximo, en cada dimensión, para cada grupo participante. Puede observarse que los tres grupos muestran perfiles dimensionales similares, siendo la dimensión solución de problemas la que logra el mayor puntaje y se diferencia significativamente de las otras de acuerdo a los resultados del ANOVA aplicado para identificar diferencias entre las dimensiones del test en cada grupo. 


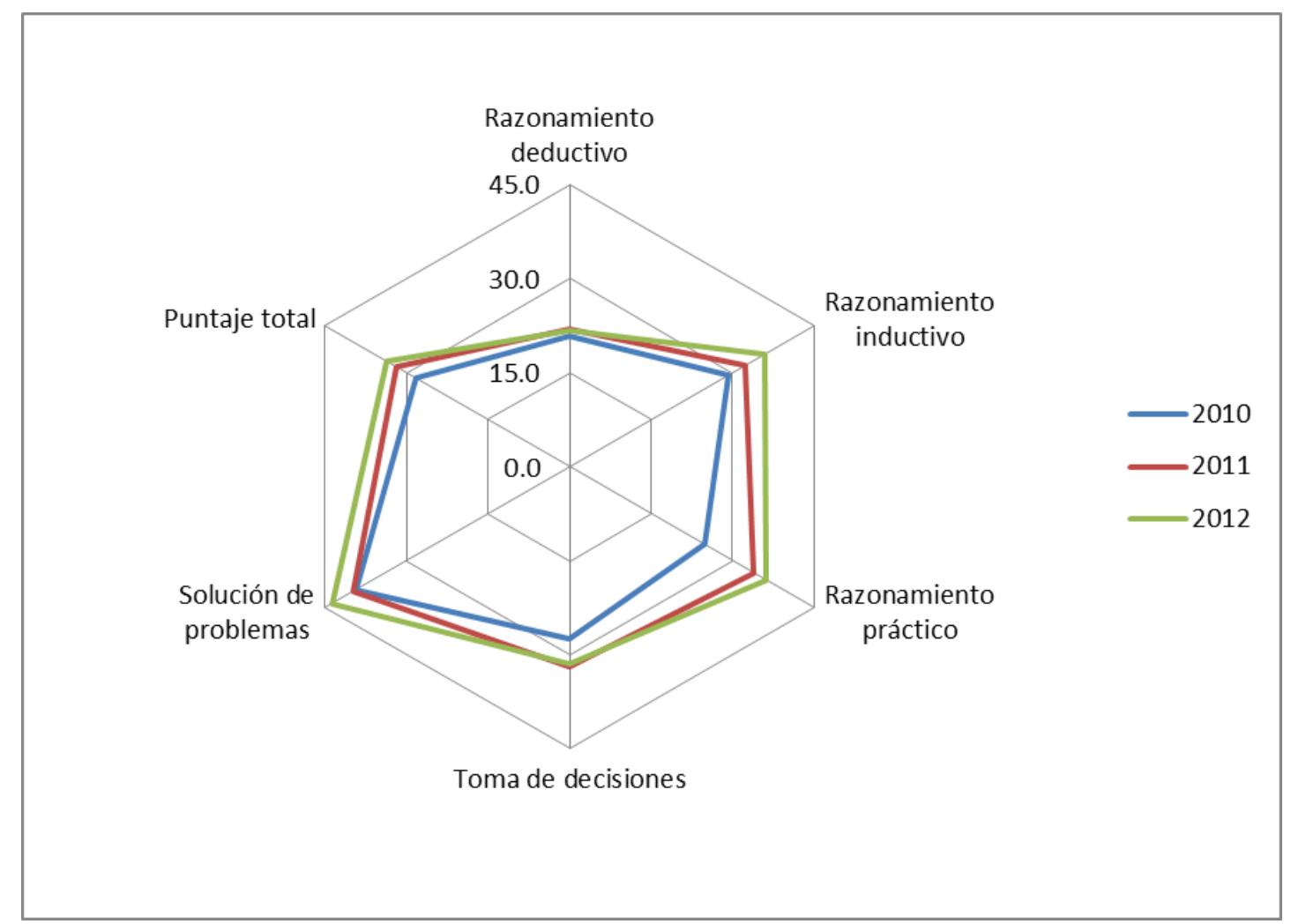

Figura 5. Resultados obtenidos en el test PENCRISAL por los tres grupos participantes: $2010(\mathrm{~N}=48)$, $2011(\mathrm{~N}=49), 2012 \mathrm{~N}=57)$

En un segundo estudio (Morales Bueno, Rivas \& Saiz, 2017), se comparó los resultados obtenidos para el grupo 2012 con los de uno de los grupos participantes del programa ARDESOS de la U. de Salamanca. Como se ha señalado, este es un programa de enseñanza directa de habilidades de pensamiento crítico. El grupo español tenía una edad promedio de 19 años y su composición era $80.3 \%$ varones y $19.7 \%$ mujeres. Se comparó las diferencias entre los puntajes de post y pre-test obtenidos con el PENCRISAL en ambos grupos. En la Figura 6 se muestran los resultados.

Se puede observar que las diferencias entre post y pre-test fueron mayores para el grupo ARDESOS en las dimensiones razonamiento deductivo, razonamiento practico, toma de decisiones y en el puntaje total. El grupo ABP híbrido superó al primero únicamente en la dimensión solución de problemas. Todas las diferencias mencionadas fueron significativas.

Los resultados obtenidos sustentan lo que se ha venido discutiendo a lo largo de este trabajo, que la enseñanza intencionada y explícita de habilidades de pensamiento crítico permite alcanzar mayores niveles de logro. En el caso de las modalidades híbridas ABP, por lo general se da prioridad al aprendizaje relacionado con el contenido y con el desarrollo de algunas habilidades como las de trabajo en equipo, búsqueda de información y solución de problemas. Las habilidades de pensamiento crítico se trabajan de manera indirecta por lo que no se puede asegurar que tengan el desarrollo esperado en el marco de un proceso ABP. 


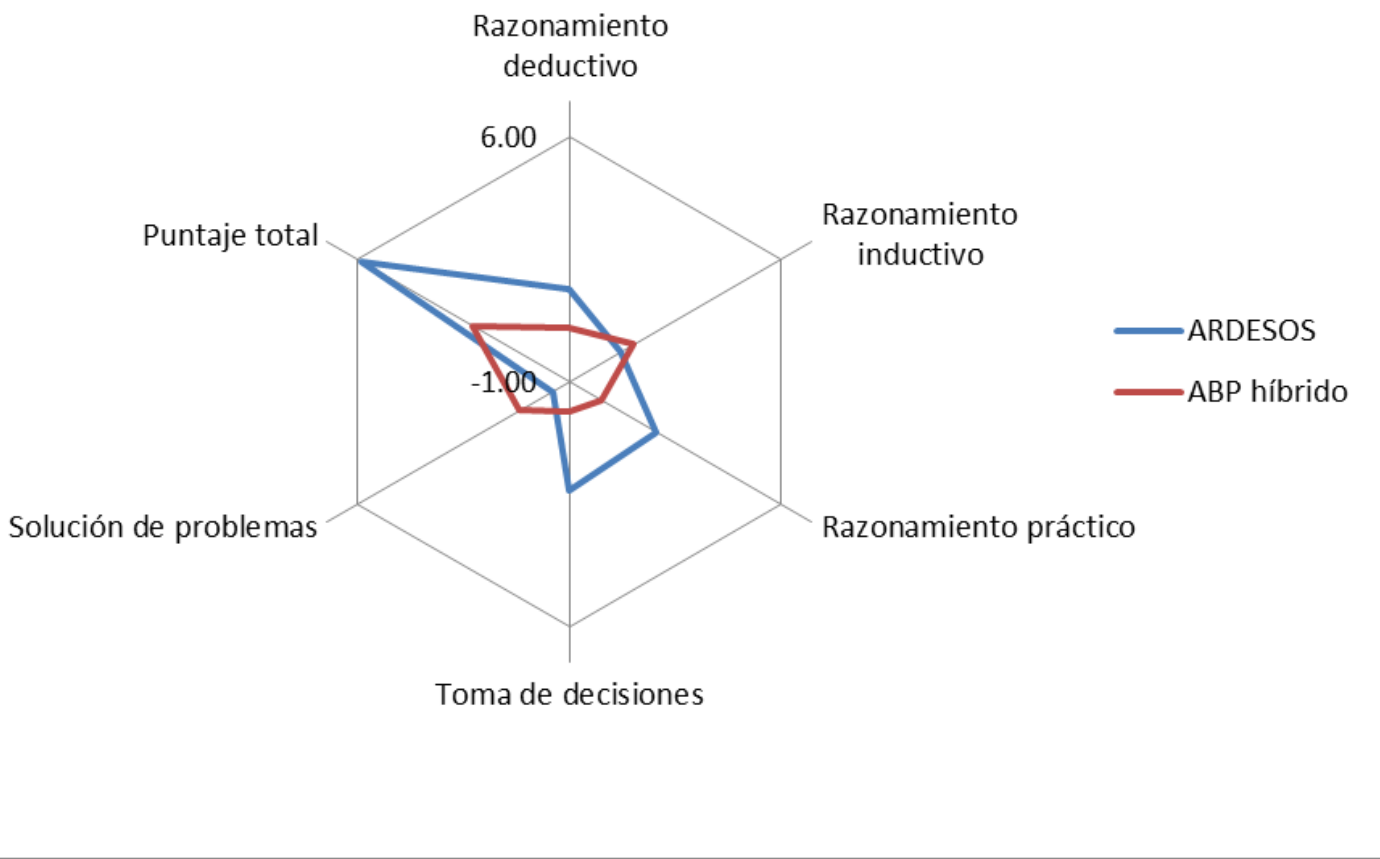

Figura 6. Resultados obtenidos para las diferencias entre post y pre-test con el PENCRISAL por los grupos ARDESOS $(\mathrm{N}=61)$ y ABP híbrido $2012(\mathrm{~N}=57)$

\section{Conclusiones}

La interrogante que ha guiado el desarrollo de este trabajo se ha enfocado en poner en evidencia los puntos de encuentro de las características de la metodología ABP y de las dimensiones de la enseñanza de habilidades de pensamiento. A lo largo de la discusión realizada ha quedado claro que existen aspectos bastantes sensibles que pueden contribuir, o no, a que estos puntos de encuentro se conviertan en espacios en donde se establezca relaciones vinculantes entre ellos y que redunden en la mejora del pensamiento crítico de los estudiantes.

Un primer punto es la necesidad de que la enseñanza de habilidades de pensamiento se ubique en un contexto educativo centrado en el estudiante, en el que se priorice su actividad durante el proceso de aprendizaje. Sin embargo, esto no debe entenderse como un simple incremento del activismo en la práctica pedagógica. La actividad implicada en una metodología como el ABP refiere al uso consciente y reflexivo de las habilidades de pensamiento, además del despliegue y desarrollo de habilidades para la investigación, la comunicación y el trabajo en equipo. En consecuencia, la planificación de la enseñanza debe incluir como meta el aprendizaje y desarrollo del pensamiento de orden superior, además del aprendizaje de contenidos y de estrategias para la solución de problemas.

El escenario o problema $A B P$ es uno de los componentes críticos a considerar en esta intención. Como se ha descrito, es deseable que en el diseño del escenario exista una articulación armoniosa entre los componentes centrales (contenido, contexto y conexión) y los componentes vinculados estrechamente con las habilidades de razonamiento, investigación y reflexión. En este sentido, el nivel de complejidad y desestructuración va de la mano con el grado de autonomía que puede disponer el estudiante para involucrarse en 
la búsqueda de propuestas de solución al problema. Sin embargo, un buen diseño debe complementarse con un proceso en donde la labor del facilitador para modelar el buen uso de habilidades de razonamiento es fundamental. Como se ha señalado, el aprendizaje y la práctica consciente de habilidades de pensamiento de orden superior deben formar parte de la planificación de la enseñanza y como tal, también de su evaluación.

Las Ilamadas modalidades híbridas ABP son bastante frecuentes en la enseñanza universitaria, principalmente porque el cambio conceptual que requiere una transformación radical de la educación superior es bastante difícil. La mayor parte de experiencias reportadas se realiza en asignaturas independientes en un contexto curricular tradicional. Esta situación trae normalmente como consecuencia que el diseño metodológico tenga que adaptarse a las condiciones particulares de la implementación. Esto es, hay que ceñirse a contenidos limitados por la disciplina, a los tiempos establecidos por el currículo y el plan de estudios, al sistema de evaluación institucional y, a otros aspectos como pueden ser los recursos logísticos, de acceso a información, etc. ¿Cuáles son las principales consecuencias para el diseño ABP?

Es común que los escenarios de las modalidades híbridas sacrifiquen el carácter integrador y holístico de las modalidades puras, ya que deben restringirse a objetivos de aprendizaje acotados. Ello implica que tengan menor complejidad y mayor estructuración, dado que su desarrollo debe ajustarse a un tiempo establecido. El grado de autonomía del que los estudiantes pueden disponer es moderado. Si bien, se rescatan los componentes de contenido, contextualización y conexión, son los componentes más cercanos al uso y desarrollo de habilidades de razonamiento los que terminan por inhibirse en el desarrollo del diseño y del proceso. De esta manera, el principal interés de los docentes es verificar el aprendizaje de los contenidos y de algunas habilidades, como la de solución de problemas $y$, en consecuencia, la evaluación se centra en estos aspectos.

Los resultados de investigación que se han reportado aquí, constituyen un referente importante que confirma lo que se viene discutiendo. Un modelo híbrido en el que se reconoce un grado moderado de control del docente, así como una delimitación del contenido en una asignatura y una estructuración de moderada a alta, reduce considerablemente las oportunidades para que se establezca una relación vinculante con el desarrollo de habilidades de pensamiento crítico. Al tratarse de un modelo dependiente de un dominio como la ciencia, es explicable que las habilidades con logro significativo sean las de solución de problemas.

En el caso de los resultados obtenidos con el programa ARDESOS, la situación es diferente. Al tratarse de un modelo de enseñanza directa de habilidades de pensamiento que se desarrolla a través de la metodología ABP, en el que se otorga un alto grado de autonomía al estudiante, se propician los espacios para que se establezca la relación vinculante entre el modelo aplicado y el desarrollo de habilidades de pensamiento crítico.

Si bien el programa ARDESOS es uno de los modelos propuestos para la enseñanza de habilidades de pensamiento independiente de un dominio, se puede tomar como referencia para mejorar el diseño de modelos híbridos desarrollados en módulos o asignaturas específicas. Es necesario considerar los aspectos sensibles que se han puesto de manifiesto en este trabajo: la complejidad y estructuración del escenario, la incorporación intencionada de oportunidades para el uso y desarrollo de habilidades de pensamiento, adecuando la participación del docente en función del nivel de avance que muestren los estudiantes. Para ello, se hace necesario incluir la evaluación de estos aprendizajes como parte del diseño pedagógico, tanto para el monitoreo como para la verificación de logros. De esta manera se 
tendría mayor coherencia entre las metas de la implementación de una aproximación al ABP y los logros obtenidos.

La importancia de la enseñanza de habilidades de pensamiento de orden superior, como lo son las de pensamiento crítico, es reconocida en todos los niveles educativos. Sin embargo, aún no se ha reportado suficiente evidencia de logros en el desarrollo de estas habilidades, particularmente en la educación universitaria. La metodología ABP es una opción atractiva, pero siempre que se tomen en cuenta los aspectos más sensibles, discutidos en este trabajo, en el diseño del problema y del proceso para facilitar el aprendizaje y fortalecimiento de estas habilidades en los estudiantes.

\section{Referencias}

Albaaly, I. (2012). The effectiveness of using Cyclic Inquiry Model (CIM) in developing some of science processes and the achievement in Science. Journal of Educational Research, 31(26), 259-283.

Barrows, H. (1996). Problem-based learning in medicine and beyond: a brief overview. En L. Wilkerson, W. H. Gijselaers (Eds.), Bringing problem-based learning to higher education: theory and practice (pp. 3-12). San Francisco: Jossey-Bass Inc. Publishers.

Biggs, J. (2005). Calidad del aprendizaje universitario. Madrid: Narcea S. A. de ediciones.

Boud, D. y Feletti, G. (Eds.) (1997). The challenge of problem-based learning, 2nd ed. London: Kogan Page Limited.

Duch, B.; Groh, S. y Allen, D. (Eds.) (2001). The power of problem-based learning. Virginia: Stylus Publishing, LLC.

Engel, C. (1997). Not just a method but a way of learning. En D. Boud y G. Feletti (Eds.), The challenge of problem-based learning (2a Ed.), (pp. 17-27). Londres: Kogan Page Limited.

Ennis, R. (1996). Critical thinking. N.J: Prentice-Hall.

Facione, P. A. (2000). The disposition toward critical thinking: its character, measurement, and relationship to critical thinking skill, Informal Logic, 20(1), 61-84.

Facione, P. A. (2007). Critical thinking: What it is and why it counts. Recuperado el 20 de abril de 2016, de: www.insightassessment.com

Facione, P. A. (2011). Think critically, New York: Prentice-Hall.

Gijbels, D.; Dochy, F.; Van den Bossche, P. y Segers, M. (2005). Effects of problem-based learning: a meta-analysis from the angle of assessment. Review of Educational Research, 75(1), 27-61.

Graaff, E. de, \& Kolmos, A. (2003). Characteristics of problem-based learning. International Journal of Engineering Education, 5(19), 657-662.

Guerra, A., \& Kolmos, A. (2011). Comparing problem-based learning models: suggestions for their implementation. In J. Davies, E. de Graaff \& A. Kolmos (Eds.), PBL across the disciplines: research into the best practice (pp. 3-16). Aalborguniversitestforlag, Aalborg. 
Halpern, D. (1989). Thought and knowledge. An introduction to critical thinking, 2nd ed. New Jersey: Lawrence Erlbaum Associates, Publishers.

Halpern, D. (1998). Teaching critical thinking for transfer across domains. Dispositions, skills, structure training, and metacognitive monitoring. American Psychologist, 53(4), 449455 .

Hmelo-Silver, C. E. (2004). Problem-Based Learning: What and how do students learn? Educational Psychology Review, 16(3), 235-266.

Hmelo-Silver, C. \& Barrows, H. (2006). Goals and strategies of a problem-based learning facilitator. The Interdisciplinary Journal of Problem-based Learning, 1(1), 21-39.

Hmelo-Silver, C. E., \& Ferrari, M. (1997). The Problem-Based Learning tutorial: Cultivating Higher Order Thinking Skills. Journal for the Education of the Gifted, 20(4), 401-422.

Hung, W. (2006). The 3C3R model: a conceptual framework for designing problems in PBL. Interdisciplinary Journal of Problem-based Learning. (1) 1, 55-77.

Margetson, D. (1997). Why is problem-based learning a challenge? En D. Boud y G. Feletti (Eds.), The challenge of problem-based learning (2a Ed.), (pp. 36-44). Londres: Kogan Page Limited.

Marshall, J. C., \& Horton, R. M. (2011). The Relationship of Teacher-Facilitated, Inquiry-Based Instruction to Student Higher-Order Thinking. School Science and Mathematics, 111(3), 93-101.

Miri, B., David, B.-C., \& Uri, Z. (2007). Purposely teaching for the promotion of higher-order thinking skills: A case of critical thinking. Research in Science Education, 37(4), 353369.

Morales Bueno P. (2016). Evolution of the application of an educational innovation in a general chemistry course. International Conference New Perspectives in Science Education, 5th edition, Florence, Italy, 17-18 march, Conference Proceedings, Pixel Ed., Italia: Libreriauniversitaria.it, 660-665.

Morales Bueno P., Rivas, S. F. \& Saiz, C. (2017). Estudio comparativo de desarrollo de habilidades de pensamiento crítico: ARDESOS versus ABP. III Seminario Internacional de Pensamiento Crítico, Manizales, Colombia, 11-13 octubre, Conference Proceedings, Universidad de Caldas.

Newmann, F. M. (1990). Higher order thinking in teaching social studies: A rationale for the assessment of classroom thoughtfulness. Journal of Curriculum Studies, 22(1), 41-56.

Phan, H. P. (2009). Exploring students' reflective thinking practice, deep processing strategies, effort, and achievement goal orientations. Educational Psychology, 29(3), 297-313. doi: 10.1080/01443410902877988.

Rivas, S. F. y Saiz, C. (2012). Validación y propiedades psicométricas de la prueba de pensamiento crítico PENCRISAL. Revista Electrónica de Metodología Aplicada, 17(1), 18-34.

Rivas, S. F., Morales Bueno, P. y Saiz, C. (2014). Propiedades psicométricas de la adaptación peruana de la prueba de pensamiento crítico PENCRISAL, Avaliação Psicológica, 13(2), 257-268.

Saiz, C. y Rivas, S. F. (2011). Evaluation of the ARDESOS program: an initiative to improve critical thinking skills. Journal of the Scholarship of Teaching and Learning, 11(2), 34-51. 
Saiz, C. y Rivas, S. F. (2012). Pensamiento crítico y aprendizaje basado en problemas. Revista de Docencia Universitaria, 10(3), 325-346.

Savery, J. (2006). Overview of problem-based learning: definitions and distinctions. The Interdisciplinary Journal of Problem-based Learning, 1(1), 9-20.

Savin-Baden, M. (2000). Problem-based learning in higher education: Untold stories. Buckingham: SRHE and Open University Press.

Snyder, L. G., \& Snyder, M. J. (2008). Teaching critical thinking and problem solving skills. The Delta Pi Epsilon Journal, L(2), 90-99.

Walton, D. (2006). Fundamentals of critical argumentation. UK: Cambridge University Press.

Wilkerson, L., \& Gijselaers, W. (Eds.) (1996). Bringing problem-based learning to higher education: theory and practice. San Francisco: Jossey-Bass Inc. Publishers. 